\title{
E-Mail Compliance - Wunsch und Wirklichkeit
}

Das Erscheinen dieses DuD-Hefts wird von zahlreichen Aktivitäten begleitet, die sich um die Weiterentwicklung der E-Mail als Internet Basisanwendung bemühen. Deren IETF-Sicherheitsstandard S/MIME stammt aus dem Jahr 1995 und ist wie PGP auf eine Ende-zu-Ende Anwendung von Verschlüsselung und Signaturen durch den Nutzer ausgelegt. Weil die E-Mail Kommunikation inzwischen breite geschäftliche Anwendung mit verbindlichen Folgen gefunden hat, haben sich die Bewertung ihrer Zulässigkeit und die zu erfüllenden qualitativen Eigenschaften grundlegend gewandelt.

Dass die Basisstandards letztlich nicht erfolgreich sind, zeigt ihre geringe Verbreitung:Trotz vielfältiger (und auch allgemein bekannter) Möglichkeiten, den Mailverkehr im Internet mitzulesen, sind heutzutage immer noch weniger als $4 \%$ aller E-Mails verschlüsselt. Doch kaum eine andere Internet-Anwendung erfreut sich einer vergleichbaren Akzeptanz und Verbreitung, ungeachtet der mangelhaften Sicherheit, und das nicht nur im Punkto Vertraulichkeit!

Im Zusammenhang mit geschäftlichen Anwendungen hat sich das Anforderungsbild entscheidend verändert. Zur E-Mail Compliance gehören nun die IT-Sicherheit mit den Hauptaspekten Vertraulichkeit, Integrität, Verbindlichkeit und Verfügbarkeit, aber auch Datenaufbewahrung und Datenschutz. Unternehmen unterliegen zahlreichen rechtlichen Verpflichtungen, deren Nichteinhaltung zu Geldstrafen und Haftungsverpflichtungen führen kann.

Die Anforderungen an einen vertrauenswürdigen E-Mail-Dienst, der "alle“ Compliance-Anforderungen erfüllt, sind sehr vielfältig und komplex. Die Einhaltung von EMail-Standards, eine starke Identifizierung und Authentisierung, die Nutzung von Verschlüsselung, die Verwendung von digitalen Signaturen, eine angemessene Archivierung von E-Mails, der richtige Umgang mit E-Mails und E-Mail-Adressen sowie Logdaten, der passende Umgang mit Spams, Malware, Phishing-Angriffe sind einige Herausforderungen, die es zu bewältigen gilt.

In dieser Ausgabe der DuD werden Aspekte von „E-Mail Compliance“ von verschiedenen Standpunkten und unterschiedlichen Qualitäten sowie Zielrichtungen beleuchtet.

In einer Zeit, in der die (deutsche) Post das „Briefgeheimnis" ins Internet bringen will und De-Mail gesetzlich vorbereitet wird, ist die Zeit gekommen, über E-Mail 2.0 zu diskutieren, um einen international nutzbaren, vertrauenswürdigen E-Mail-Dienst zu etablieren.

Zu dieser wichtigen Diskussion sollen die folgenden Artikel anregen.

Zunächst beleuchtet Norbert PohImann die Risiken des realen E-Mail-Dienstes und erläutert verfügbare Gegenmaßnahmen.

Mit der nach wie vor kontrovers diskutierten Frage der privaten E-Mail-Nutzung in Organisationen setzen sich Sven Polenz und Sven Thomsen auseinander.

Eine der komplexesten Aufgaben im E-Mail Compliance-Spektrum ist die Archivierung. Ulrich Kampffmeyer vermittelt einen fundierten Überblick zum Stand der Dinge.

Immer noch eine Baustelle: Rund um die E-Mail-Verschlüsselung geht es im Beitrag von Thomas Hemker.

Wilfried Reiners erläutert, warum eine E-Mail Richtlinie in Organisationen unverzichtbar ist.

Unter welchen Bedingungen E-Mails den Anforderungen eines elektronischen Rechtsverkehrs erfüllen, zeigt Paul E. Mertes auf.

Schließlich werden in den Beiträgen von Ekkehard Diedrich und Dominik Birk / Christoph Wegener Infrastrukturaspekte erläutert, die im Zusammenhang mit E-Mail-Anwendungen zur Verfügung stehen.

Ein Bericht aus der Sicht der E-Mail-Provider - GMX, WEB.DE und der Privatkundensparte der Deutschen Telekom - zum Ergebnis des De-Mail Pilotprojektes in Friedrichshafen bildet den Abschluss des Schwerpunktes dieses DuD Heftes.

Wir wünschen den Leserinnen und Lesern viele Erkenntnisse und Anregungen bei der Beschäftigung mit dem wichtigen Thema E-Mail-Compliance. 